\title{
Locally Decodable and Updatable Non-malleable Codes and Their Applications
}

\author{
Dana Dachman-Soled ${ }^{1}$, Feng-Hao Liu ${ }^{2}$, Elaine Shi ${ }^{2}$, and Hong-Sheng Zhou ${ }^{3}$ \\ 1 University of Maryland, USA \\ danadach@ece.umd.edu \\ 2 University of Maryland, USA \\ \{fenghao, elaine $\}$ cs. umd.edu \\ 3 Virginia Commonwealth University, USA \\ hszhou@vcu.edu
}

\begin{abstract}
Non-malleable codes, introduced as a relaxation of errorcorrecting codes by Dziembowski, Pietrzak and Wichs (ICS '10), provide the security guarantee that the message contained in a tampered codeword is either the same as the original message or is set to an unrelated value. Various applications of non-malleable codes have been discovered, and one of the most significant applications among these is the connection with tamper-resilient cryptography. There is a large body of work considering security against various classes of tampering functions, as well as non-malleable codes with enhanced features such as leakage resilience.

In this work, we propose combining the concepts of non-malleability, leakage resilience, and locality in a coding scheme. The contribution of this work is three-fold:

1. As a conceptual contribution, we define a new notion of locally decodable and updatable non-malleable code that combines the above properties.

2. We present two simple and efficient constructions achieving our new notion with different levels of security.

3. We present an important application of our new tool-securing RAM computation against memory tampering and leakage attacks. This is analogous to the usage of traditional non-malleable codes to secure implementations in the circuit model against memory tampering and leakage attacks.
\end{abstract}

\section{Introduction}

The notion of non-malleable codes was defined by Dziembowski, Pietrzak and Wichs 22 as a relaxation of error-correcting codes. Informally, a coding scheme is non-malleable against a tampering function if by tampering with the codeword, the function can either keep the underlying message unchanged or change it to an unrelated message. Designing non-malleable codes is not only an interesting mathematical task, but also has important implications in cryptography; for example, Coretti et al. 11] showed an efficient construction of a mulit-bit CCA 
secure encryption scheme from a single-bit one via non-malleable codes. Agrawal et al. 3. showed how to use non-malleable codes to build non-malleable commitments. Most notably, the notion has a deep connection with security against socalled physical attacks; indeed, using non-malleable codes to achieve security against physical attacks was the original motivation of the work [22]. Due to this important application, research on non-malleable codes has become an important agenda, and drawn much attention in both coding theory and cryptography.

Briefly speaking, physical attacks target implementations of cryptographic algorithms beyond their input/output behavior. For example, researchers have demonstrated that leaking/tampering with sensitive secrets such as cryptographic keys, through timing channel, differential power analysis, and various other attacks, can be devastating [2, 4, 5, 32, 39, 40, 45], and therefore the community has focused on developing new mechanisms to defend against such strong adversaries 12, 13, 15, 19, 21, 26, 28, 30, 31, 34, 36, 38, 43, 44, 46. Dziembowski, Pietrzak and Wichs 22 showed a simple and elegant mechanism to secure implementations against memory tampering attacks by using non-malleable codes - instead of storing the secret (in the clear) on a device, one instead stores an encoding of the secret. The security of the non-malleable code guarantees that the adversary cannot learn more than what can be learnt via black box access to the device, even though the adversary may tamper with memory.

In a subsequent work, Liu and Lysyanskaya 42 extended the notion to capture leakage resilience as well - in addition to non-malleability, the adversary cannot learn anything about the underlying message even while obtaining partial leakage of the codeword. By using the approach outlined above, one can achieve security guarantees against both tampering and leakage attacks. In recent years, researchers have been studying various flavors of non-malleable codes; for example some work has focused on constructions against different classes of tampering functions, some has focused on different additional features, (e.g. continual attacks, rates of the scheme, etc), and some focused on other applications $[1,3,7,9,20,23,25]$.

In this paper, we focus on another important feature inspired from the field of coding theory - locality. More concretely, we consider a coding scheme that is locally decodable and updatable. As introduced by Katz and Trevisan [37], local decodability means that in order to retrieve a portion of the underlying message, one does not need to read through the whole codeword. Instead, one can just read a few locations at the codeword. Similarly, local updatability means that in order to update some part of the underlying messages, one only needs to update some parts of the codeword. Locally decodable codes have many important applications in private information retrieval [10] and secure multi-party computation [33, and have deep connections with complexity theory; see [49. Achieving local decodability and updatability simultaneously makes the task more challenging. Recently, Chandran et al. 6] constructed a locally decodable and updatable code in the setting of error-correcting codes. They also show an application to dynamic proofs of retrievability. Motivated by the above results, we further ask the following intriguing question: 
Can we build a coding scheme enjoying all three properties, i.e., nonmalleability, leakage resilience, and locality? If so, what are its implications in cryptography?

Our Results. In light of the above questions, our contribution is three-fold:

- (Notions). We propose new notions that combine the concepts of nonmalleability, leakage resilience, and locality in codes. First, we formalize a new notion of locally decodable and updatable non-malleable codes (against one-time attacks). Then, we extend this new notion to capture leakage resilience under continual attacks.

- (Constructions). We present two simple constructions achieving our new notions. The first construction is highly efficient - in order to decode (update) one block of the encoded messages, only two blocks of the codeword must be read (written) - but is only secure against one-time attacks. The second construction achieves security against continual attacks, while requiring $\log (n)$ number of reads (writes) to perform one decode (update) operation, where $n$ is the number of blocks of the underlying message.

- (Application). We present an important application of our new notion - achieving tamper and leakage resilience in the random access machine (RAM) model. We first define a new model that captures tampering and leakage attacks in the RAM model, and then give a generic compiler that uses our new notion as a main ingredient. The compiled machine will be resilient to leakage and tampering on the random access memory. This is analogous to the usage of traditional non-malleable codes to secure implementations in the circuit model.

\subsection{Techniques}

In this section, we present a technical overview of our results.

Locally Decodable Non-malleable Codes. Our first goal is to consider a combination of concepts of non-malleability and local decodability. Recall that a coding scheme is non-malleable with respect to a tampering function $f$ if the decoding of the tampered codeword remains the same or becomes some unrelated message. To capture this idea, the definition in the work 22] requires that there exists a simulator (with respect to such $f$ ) who outputs same* if the decoding of the tampered codeword remains the same as the original one, or he outputs a decoded message, which is unrelated to the original one. In the setting of local decodability, we consider encodings of blocks of messages $M=\left(m_{1}, m_{2}, \ldots, m_{n}\right)$, and we are able to retrieve $m_{i}$ by running $\operatorname{DEC}^{\operatorname{ENC}(M)}(i)$, where the decoding algorithm gets oracle access to the codeword.

The combination faces a subtlety that we cannot directly use the previous definition: suppose a tampering function $f$ only modifies one block of the codeword, then it is likely that DEC remains unchanged for most places. (Recall a DEC will only read a few blocks of the codeword, so it may not detect the modification.) 
In this case, the (overall) decoding of $f(C)$ (i.e. $\left.\left(\operatorname{DEC}^{f(C)}(1), \ldots, \operatorname{DEC}^{f(C)}(n)\right)\right)$ can be highly related to the original message, which intuitively means it is highly malleable.

To handle this issue, we consider a more fine-grained experiment. Informally, we require that for any tampering function $f$ (within some class), there exists a simulator that computes a vector of decoded messages $\boldsymbol{m}^{*}$, a set of indices $\mathcal{I} \subseteq[n]$. Here $\mathcal{I}$ denotes the coordinates of the underlying messages that have been tampered with. If $\mathcal{I}=[n]$, then the simulator thinks that the decoded messages are $\boldsymbol{m}^{*}$, which should be unrelated to the original messages. On the other hand, if $\mathcal{I} \subsetneq[n]$, the simulator thinks that all the messages not in $\mathcal{I}$ remain unchanged, while those in $\mathcal{I}$ become $\perp$. This intuitively means the tampering function can do only one of the following cases:

1. It destroys a block (or blocks) of the underlying messages while keeping the other blocks unchanged, or

2. If it modifies a block of the underlying messages to some unrelated string, then it must have modified all blocks of the underlying messages to encodings of unrelated messages.

Our construction of locally decodable non-malleable code is simple - we use the idea similar to the key encapsulation mechanism/data encapsulation mechanism (KEM/DEM) framework. Let NMC be a regular non-malleable code, and $\mathcal{E}$ be a secure (symmetric-key) authenticated encryption. Then to encode blocks of messages $M=\left(m_{1}, \ldots, m_{n}\right)$, we first sample a secret key sk of $\mathcal{E}$, and then output $\left(\mathrm{NMC} . \operatorname{ENC}(\mathrm{sk}), \mathcal{E}\right.$.Encrypt sk $\left(m_{1}, 1\right), \ldots, \mathcal{E}$.Encrypt sk $\left.\left(m_{n}, n\right)\right)$. The intuition is clear: if the tampering function does not change the first block, then by security of the authenticated encryption, any modification of the rest will become $\perp$. (Note that here we include a tag of positions to prevent permutation attacks). On the other hand, if the tampering function modified the first block, it must be decoded to an unrelated secret key sk'. Then by semantic security of the encryption scheme, the decoded values of the rest must be unrelated. The code can be updated locally: in order to update $m_{i}$ to some $m_{i}^{\prime}$, one just need to retrieve the $1^{\text {st }}$ and $(i+1)^{\text {st }}$ blocks. Then he just computes a fresh encoding of NMC.ENC(sk) and the ciphertext $\mathcal{E}$. Encrypt ${ }_{\text {sk }}\left(m_{i}^{\prime}\right)$, and writes back to the same positions.

Extensions to Leakage Resilience against Continual Attacks. We further consider a notion that captures leakage attacks in the continual model. First we observe that suppose the underlying non-malleable code is also leakage resilient 42, the above construction also achieves one-time leakage resilience. Using the same argument of Liu and Lysyanskaya 42, if we can refresh the whole encoding, we can show that the construction is secure against continual attacks. However, in our setting, refreshing the whole codeword is not counted as a solution since this is in the opposite of the spirit of our main theme - locality. The main challenge is how to refresh (update) the codeword locally while maintaining tamper and leakage resilience.

To capture the local refreshing and continual attacks, we consider a new model where there is an updater $\mathcal{U}$ who reads the whole underlying messages and 
decides how to update the codeword (using the local update algorithm). The updater is going to interact with the codeword in a continual manner, while the adversary can launch tampering and leakage attacks between two updates. To define security we require that the adversary cannot learn anything of the underlying messages via tampering and leakage attacks from the interaction.

We note that if there is no update procedure at all, then no coding scheme can be secure against continual leakage attacks if the adversary can learn the whole codeword bit-by-bit. In our model, the updater and the adversary take turns interacting with the codeword - the adversary tampers with and/or gets leakage of the codeword, and then the updater locally updates the codeword, and the process repeats. See Section 2 for the formal model.

Then we consider how to achieve this notion. First we observe that the construction above is not secure under continual attacks: suppose by leakage the adversary can get a full ciphertext $\mathcal{E}$.Encrypt sk $\left(m_{i}, i\right)$ at some point, and then the updater updates the underlying message to $m_{i}^{\prime}$. In the next round, the adversary can apply a rewind attack that modifies the codeword back with the old ciphertext. Under such attack, the underlying messages have been modified to some related messages. Thus the construction is not secure.

One way to handle this type of rewind attacks is to tie all the blocks of ciphertexts together with a "time stamp" that prevents the adversary from replacing the codeword with old ciphertexts obtained from leakage. A straightforward way is to hash all the blocks of encryptions using a collision resistant hash function and also encode this value into the non-malleable code, i.e., $C=\left(\operatorname{NMC} . \operatorname{EnC}(\right.$ sk, $v), \mathcal{E}$.Encrypt $\left(1, m_{1}\right), \ldots, \mathcal{E}$.Encrypt $\left.\left(n, m_{n}\right)\right)$, where $v=$ $h\left(\mathcal{E}\right.$.Encrypt $\left(1, m_{1}\right), \ldots, \mathcal{E}$. Encrypt $\left.\left(n, m_{n}\right)\right)$. Intuitively, suppose the adversary replaces a block $\mathcal{E}$. Encrypt $\left(i, m_{i}\right)$ by some old ciphertexts, then it would be caught by the hash value $v$ unless he tampered with the non-malleable code as well. But if he tampers with the non-malleable code, the decoding will be unrelated to sk, and thus the rest of ciphertexts become "un-decryptable". This approach prevents the rewind attacks, yet it does not preserve the local properties, i.e. to decode a block, one needs to check the consistency of the hash value $v$, which needs to read all the blocks of encryptions. To prevent the rewind attacks while maintaining local decodability/updatability, we use the Merkle tree technique, which allows local checks of consistency. The final encoding outputs the following:

$$
\left(\operatorname{NMC} . \operatorname{EnC}(\mathrm{sk}, v), \mathcal{E} . \operatorname{Encrypt}\left(1, m_{1}\right), \ldots, \mathcal{E} \text {.Encrypt }\left(n, m_{n}\right), T\right)
$$

where $T$ is the Merkle tree of $\left(\mathcal{E}\right.$.Encrypt $\left(1, m_{1}\right), \ldots, \mathcal{E}$. Encrypt $\left.\left(n, m_{n}\right)\right)$, and $v$ is its root (it can also be viewed as a hash value). To decode a position $i$, the algorithm reads the $1^{\text {st }}$, and the $(i+1)^{\text {st }}$ blocks together with a path in the tree. If the path is inconsistent with the root, then output $\perp$. To update, one only needs to re-encode the first block with a new root, and update the $(i+1)^{\text {st }}$ block and the tree. We note that Merkle tree allows local updates: if there is only one single change at a leaf, then one can compute the new root given only a path passing through the leaf and the root. So the update of the codeword can be 
done locally by reading the $1^{\text {st }}$, the $(i+1)^{\text {st }}$ blocks and the path. We provide a detailed description and analysis in Section 3.2.

Concrete Instantiations. In our construction above, we rely on an underlying non-malleable code NMC against some class of tampering functions $\mathcal{F}$ and leakage resilient against some class of leakage functions $\mathcal{G}$. The resulting encoding scheme is a locally decodable and updatable coding scheme which is continual non-malleable against some class $\overline{\mathcal{F}}$ of tampering functions and leakage resilient against some class $\overline{\mathcal{G}}$ of leakage functions, where the class $\overline{\mathcal{F}}$ is determined by $\mathcal{F}$ and the class $\overline{\mathcal{G}}$ is determined by $\mathcal{G}$. In order to understand the relationship between these classes, it is helpful to recall the structure of the output of the final encoding scheme. The final encoding scheme will output $2 n+1$ blocks $x_{1}, \ldots, x_{2 n+1}$ such that the first block $x_{1}$ is encoded using the underlying non-malleable code NMC. As a first attempt, we can define $\overline{\mathcal{F}}$ to consist of tampering functions $f\left(x_{1}, \ldots, x_{2 n+1}\right)=\left(f_{1}\left(x_{1}\right), f_{2}\left(x_{2}, \ldots, x_{2 n+1}\right)\right)$, where $f_{1} \in \mathcal{F}$ and $f_{2}$ is any polynomial-sized circuit. However, it turns out that we are resilient against an even larger class of tampering functions! This is because the tampering function $f_{1}$ can actually depend on all the values $x_{2}, \ldots, x_{2 n+1}$ of blocks $2, \ldots,(2 n+1)$. Similarly, for the class of leakage functions, as a first attempt, we can define $\overline{\mathcal{G}}$ to consist of leakage functions $g\left(x_{1}, \ldots, x_{2 n+1}\right)=$ $\left(g_{1}\left(x_{1}\right), g_{2}\left(x_{2}, \ldots, x_{2 n+1}\right)\right)$, where $g_{1} \in \mathcal{G}$ and $g_{2}$ is any polynomial-sized circuit. However, again we can achieve even more because the tampering function $g_{1}$ can actually depend on all the values $x_{2}, \ldots, x_{2 n+1}$. For a formal definition of the classes of tampering and leakage functions that we handle, see Theorem 3 .

Finally, we give a concrete example of what the resulting classes look like using the NMC construction of Liu and Lysyanskaya 42 as the building block. Recall that their construction achieves both tamper and leakage resilience for split-state functions. Thus, the overall tampering function $f$ restricted in the first block (i.e. $f_{1}$ ) can be any (poly-sized) split-state function. On the other hand $f$ restricted in the rest (i.e. $f_{2}$ ) can be any poly-sized function. The overall leakage function $g$ restricted in the first block (i.e. $g_{1}$ ) can be a (poly-sized) length-bounded split-state function; $g$ on the other hand, can leak all the other parts. See Section 3.3 for more details.

Application to Tamper and Leakage Resilient RAM Model. Whereas regular non-malleable codes yield secure implementations against memory tampering in the circuit model, our new tool yields secure implementations against memory tampering (and leakage) in the RAM model.

In our RAM model, the data and program to be executed are stored in the random access memory. Through a CPU with a small number of (non-persistent) register 1 , execution proceeds in clock cycles: In each clock-cycle memory addresses

\footnotetext{
${ }^{1}$ These non-persistent registers are viewed as part of the circuitry that stores some transient states while the CPU is computing at each cycle. The number of these registers is small, and the CPU needs to erase the data in order to reuse them, so they cannot be used to store a secret key that is needed for a long term of computation.
} 
are read and stored in registers, a computation is performed, and the contents of the registers are written back to memory. In our attack model, we assume that the CPU circuitry (including the non-persistent registers) is secure - the computation itself is not subject to physical attacks. On the other hand, the random access memory, and the memory addresses are prone to leakage and tampering attacks. We remark that if the CPU has secure persistent registers that store a secret key, then the problem becomes straightforward: Security can be achieved using encryption and authentication together with oblivious RAM [29]. We emphasize that in our model, persistent states of the CPU are stored in the memory, which are prone to leakage and tampering attacks. As our model allows the adversary to learn the access patterns the CPU made to the memory, together with the leakage and tampering power on the memory, the adversary can somewhat learn the messages transmitted over the bus or tamper with them (depending on the attack classes allowed on the memory). For simplicity of presentation, we do not define attacks on the bus, but just remark that these attacks can be implicitly captured by learning the access patterns and attacking the memory2.

In our formal modeling, we consider a next instruction function $\Pi$, a database $D$ (stored in the random access memory) and an internal state (using the nonpersistent registers). The CPU will interact (i.e., read/write) with the memory based on $\Pi$, while the adversary can launch tamper and leakage attacks during the interaction.

Our compiler is very simple, given the ORAM technique and our new codes as building blocks. Informally speaking, given any next instruction function $\Pi$ and database $D$, we first use ORAM technique to transform them into a next instruction function $\widetilde{\Pi}$ and a database $\widetilde{D}$. Next, we use our local non-malleable code (ENC, DEC, UPDATE) to encode $\widetilde{D}$ into $\widehat{D}$; the compiled next instruction function $\widehat{\Pi}$ does the following: run $\widetilde{\Pi}$ to compute the next "virtual" read/write instruction, and then run the local decoding or update algorithms to perform the physical memory access.

Intuitively, the inner ORAM protects leakage of the address patterns, and the outer local non-malleable codes prevent an attacker from modifying the contents of memory to some different but related value. Since at each cycle the CPU can only read and write at a small number of locations of the memory, using regular non-malleable codes does not work. Our new notion of locally decodable and updatable non-malleable codes exactly solves these issues!

\subsection{Related Work}

Different flavors of non-malleable codes were studied [1, 3, $7,9,20,22,23,25,42$, We can use these constructions to secure implementations against memory attacks in the circuit model, and also as our building block for the locally decodable/updatable non-malleable codes. See also Section 3.3 for further exposition.

\footnotetext{
${ }^{2}$ There are some technical subtleties to simulate all leakage/tampering attacks on the values passing the bus using memory attacks (and addresses). We defer the rigorous treatment to future work.
} 
Securing circuits or CPUs against physical attacks is an important task, but out of the scope of this paper. Some partial results can be found in previous work 12, 13, 16 $19,21,26,27,30,31,34,36,38,41,43,44,46,48$.

In an independent and concurrent work, Faust et al. 24] also considered securing RAM computation against tampering and leakage attacks. We note that both their model and techniques differ considerably from ours. In the following, we highlight some of these differences. The main focus of 24] is constructing RAM compilers for keyed functions, denoted $\mathcal{G}_{\mathrm{K}}$, to allow secure RAM emulation of these functions in the presence of leakage and tampering. In contrast, our work focuses on construcing compilers that transform any dynamic RAM machine into a RAM machine secure against leakage and tampering. Due to this different perspective, our compiler explicitly utilizes an underlying ORAM compiler, while they assume that the memory access pattern of input function $\mathcal{G}$ is independent of the secret state $\mathrm{K}$ (e.g., think of $\mathcal{G}$ as the circuit representation of the function). In addition to the split-state tampering and leakage attacks considered by both papers, 24] do not assume that memory can be overwritten or erased, but require the storage of a tamper-proof program counter. With regard to techniques, they use a stronger version of non-malleable codes in the splitstate setting (called continual non-malleable codes [23) for their construction. Finally, in their construction, each memory location is encoded using an expensive non-malleable encoding scheme, while in our construction, non-malleable codes are used only for a small portion of the memory, while highly efficient symmetric key authenticated encryption is used for the remainder.

\section{Locally Decodable and Updatable Non-malleable Codes}

In this section, we first review the concepts of non-malleable (leakage resilient) codes. Then we present our new notion that combines non-malleability, leakage resilience, and locality.

\subsection{Preliminary}

Definition 1 (Coding Scheme). Let $\Sigma, \hat{\Sigma}$ be sets of strings, and $\kappa, \hat{\kappa} \in \mathbb{N}$ be some parameters. A coding scheme consists of two algorithms (ENC, DEC) with the following syntax:

- The encoding algorithm (perhaps randomized) takes input a block of message in $\Sigma$ and outputs a codeword in $\hat{\Sigma}$.

- The decoding algorithm takes input a codeword in $\hat{\Sigma}$ and outputs a block of message in $\Sigma$.

We require that for any message $m \in \Sigma, \operatorname{Pr}[\operatorname{DEC}(\operatorname{ENC}(m))=m]=1$, where the probability is taken over the choice of the encoding algorithm. In binary settings, we often set $\Sigma=\{0,1\}^{\kappa}$ and $\hat{\Sigma}=\{0,1\}^{\hat{\kappa}}$.

Definition 2 (Non-malleability [22]). Let $k$ be the security parameter, $\mathcal{F}$ be some family of functions. For each function $f \in \mathcal{F}$, and $m \in \Sigma$, define the tampering experiment: 


$$
\operatorname{Tamper}_{m}^{f} \stackrel{\text { def }}{=}\left\{\begin{array}{c}
c \leftarrow \operatorname{ENC}(m), \tilde{c}:=f(c), \tilde{m}:=\operatorname{DEC}(\tilde{c}) . \\
\text { Output }: \tilde{m} .
\end{array}\right\},
$$

where the randomness of the experiment comes from the encoding algorithm. We say a coding scheme (ENC, DEC) is non-malleable with respect to $\mathcal{F}$ if for each $f \in \mathcal{F}$, there exists a PPT simulator $\mathcal{S}$ such that for any message $m \in \Sigma$, we have

$$
\operatorname{Tamper}_{m}^{f} \approx \operatorname{Ideal}_{\mathcal{S}, m} \stackrel{\text { def }}{=}\left\{\begin{array}{c}
\tilde{m} \cup\left\{\text { same* }^{*} \leftarrow \mathcal{S}^{f(\cdot)} .\right. \\
\text { Output }: m \text { if same*; otherwise } \tilde{m} .
\end{array}\right\}
$$

Here the indistinguishability can be either computational or statistical.

We can extend the notion of non-malleability to leakage resilience (simultaneously) as the work of Liu and Lysyanskaya [42.

Definition 3 (Non-malleability and Leakage Resilience [42]). Let $k$ be the security parameter, $\mathcal{F}, \mathcal{G}$ be some families of functions. For each function $f \in \mathcal{F}, g \in \mathcal{G}$, and $m \in \Sigma$, define the tamper-leak experiment:

$$
\operatorname{TamperLeak}_{m}^{f, g} \stackrel{\text { def }}{=}\left\{\begin{array}{c}
c \leftarrow \operatorname{ENC}(m), \tilde{c}:=f(c), \tilde{m}:=\operatorname{DEC}(\tilde{c}) . \\
\text { Output }:(\tilde{m}, g(c)) .
\end{array}\right\},
$$

where the randomness of the experiment comes from the encoding algorithm. We say a coding scheme (ENC, DEC) is non-malleable and leakage resilience with respect to $\mathcal{F}$ and $\mathcal{G}$ if for any $f \in \mathcal{F}, g \in \mathcal{G}$, there exists a PPT simulator $\mathcal{S}$ such that for any message $m \in \Sigma$, we have

$\operatorname{TamperLeak}_{m}^{f, g} \approx \operatorname{Ideal}_{\mathcal{S}, m} \stackrel{\text { def }}{=}\left\{\begin{array}{c}\left(\tilde{m} \cup\left\{\text { same* }^{*}, \ell\right) \leftarrow \mathcal{S}^{f(\cdot), g(\cdot)} \cdot\right. \\ \text { Output }:(m, \ell) \text { if same*; otherwise }(\tilde{m}, \ell) .\end{array}\right\}$

Here the indistinguishability can be either computational or statistical.

\subsection{New Definitions - Codes with Local Properties}

In this section, we consider coding schemes with extra local properties - decodability and updatability. Intuitively, this gives a way to encode blocks of messages, such that in order to decode (retrieve) a single block of the messages, one only needs to read a small number of blocks of the codeword; similarly, in order to update a single block of the messages, one only needs to update a few blocks of the codeword.

Definition 4 (Locally Decodable and Updatable Code). Let $\Sigma, \hat{\Sigma}$ be sets of strings, and $n, \hat{n}, p, q$ be some parameters. An $(n, \hat{n}, p, q)$ locally decodable and updatable coding scheme consists of three algorithms

(ENC, DEC, UPDATE) with the following syntax: 
- The encoding algorithm ENC (perhaps randomized) takes input an n-block (in $\Sigma$ ) message and outputs an $\hat{n}$-block (in $\hat{\Sigma}$ ) codeword.

- The (local) decoding algorithm DEC takes input an index in [n], reads at most $p$ blocks of the codeword, and outputs a block of message in $\Sigma$. The overall decoding algorithm simply outputs $(\operatorname{DEC}(1), \operatorname{DEC}(2), \ldots, \operatorname{DEC}(n))$.

- The (local) updating algorithm UPDATE (perhaps randomized) takes inputs an index in $[n]$ and a string in $\Sigma \cup\{\epsilon\}$, and reads/writes at most $q$ blocks of the codeword. Here the string $\epsilon$ denotes the procedure of refreshing without changing anything.

Let $C \in \hat{\Sigma}^{\hat{n}}$ be a codeword. For convenience, we denote $\mathrm{DEC}^{C}$, $\mathrm{UPDATE}^{C}$ as the processes of reading/writing individual block of the codeword, i.e. the codeword oracle returns or modifies individual block upon a query. Here we view $C$ as a random access memory where the algorithms can read/write to the memory $C$ at individual different locations.

Remark 1. Throughout this paper, we only consider non-adaptive decoding and updating, which means the algorithms DEC and UPDATE compute all their queries at the same time before seeing the answers, and the computation only depends on the input $i$ (the location). In contrast, an adaptive algorithm can compute a query based on the answer from previous queries. After learning the answer to such query, then it can make another query. We leave it as an interesting open question to construct more efficient schemes using adaptive queries.

Then we define the requirements of the coding scheme.

Definition 5 (Correctness). An $(n, \hat{n}, p, q)$ locally decodable and updatable coding scheme (with respect to $\Sigma, \hat{\Sigma}$ ) satisfies the following properties. For any message $M=\left(m_{1}, m_{2}, \ldots, m_{n}\right) \in \Sigma^{n}$, let $C=\left(c_{1}, c_{2}, \ldots, c_{\hat{n}}\right) \leftarrow \operatorname{ENC}(M)$ be a codeword output by the encoding algorithm. Then we have:

- for any index $i \in[n], \operatorname{Pr}\left[\mathrm{DEC}^{C}(i)=m_{i}\right]=1$, where the probability is over the randomness of the encoding algorithm.

- for any update procedure with input $\left(j, m^{\prime}\right) \in[n] \times \Sigma \cup\{\epsilon\}$, let $C^{\prime}$ be the resulting codeword by running $\operatorname{UPDATE}^{C}\left(j, m^{\prime}\right)$. Then we have $\operatorname{Pr}\left[\operatorname{DEC}^{C^{\prime}}(j)=\right.$ $\left.m^{\prime}\right]=1$, where the probability is over the encoding and update procedures. Moreover, the decodings of the other positions remain unchanged.

Remark 2. The correctness definition can be directly extended to handle any sequence of updates.

Next, we define several flavors of security about non-malleability and leakage resilience.

One-time Non-malleability. First we consider one-time non-malleability of locally decodable codes, i.e., the adversary only tampers with the codeword once. This extends the idea of the non-malleable codes (as in Definition 2). As discussed in the introduction, we present the following definition to capture the idea that the tampering function can only do either of the following cases: 
- It destroys a block (or blocks) of the underlying messages while keeping the other blocks unchanged, or

- If it modifies a block of the underlying messages to some unrelated string, then it must have modified all blocks of the underlying messages to encodings of unrelated messages.

Definition 6 (Non-malleability of Locally Decodable Codes). An ( $n, \hat{n}$, $p, q)$-locally decodable coding scheme with respect to $\Sigma, \hat{\Sigma}$ is non-malleable against the tampering function class $\mathcal{F}$ if for all $f \in \mathcal{F}$, there exists some simulator $\mathcal{S}$ such that for any $M=\left(m_{1}, \ldots, m_{n}\right) \in \Sigma^{n}$, the experiment Tamper $_{M}^{f}$ is (computationally) indistinguishable to the following ideal experiment $\mathbf{I d e a l}_{\mathcal{S}, M}$ :

- $\left(\mathcal{I}, \boldsymbol{m}^{*}\right) \leftarrow \mathcal{S}\left(1^{k}\right)$, where $\mathcal{I} \subseteq[n], \boldsymbol{m}^{\prime} \in \Sigma^{n}$. (Intuitively $\mathcal{I}$ means the coordinates of the underlying message that have been tampered with).

- If $\mathcal{I}=[n]$, define $\boldsymbol{m}=\boldsymbol{m}^{*}$; otherwise set $\left.\boldsymbol{m}\right|_{\mathcal{I}}=\perp,\left.\boldsymbol{m}\right|_{\overline{\mathcal{I}}}=\left.M\right|_{\overline{\mathcal{I}}}$, where $\left.\boldsymbol{x}\right|_{\mathcal{I}}$ denotes the coordinates $\boldsymbol{x}[v]$ where $v \in \mathcal{I}$, and the bar denotes the complement of a set.

- The experiment outputs $\boldsymbol{m}$.

Remark 3. Here we make two remarks about the definition:

1. In the one-time security definition, we do not consider the update procedure. In the next paragraph when we define continual attacks, we will handle the update procedure explicitly.

2. One-time leakage resilience of locally decodable codes can be defined in the same way as Definition 3.

Security against Continual Attacks. In the following, we extend the security to handle continual attacks. Here we consider a third party called updater, who can read the underlying messages and decide how to update the codeword. Our model allows the adversary to learn the location that the updater updated the messages, so we also allow the simulator to learn this information. This is without loss of generality if the leakage class $\mathcal{G}$ allows it, i.e. the adversary can query some $g \in \mathcal{G}$ to figure out what location was modified. On the other hand, the updater does not tell the adversary what content was encoded of the updated messages, so the simulator needs to simulate the view without such information. We can think of the updater as an honest user interacting with the codeword (read/write). The security intuitively means that even if the adversary can launch tampering and leakage attacks when the updater is interacting with the codeword, the adversary cannot learn anything about the underlying encoded messages (or the updated messages during the interaction).

Our continual experiment consists of rounds: in each round the adversary can tamper with the codeword and get partial information. At the end of each round, the updater will run UPDATE, and the codeword will be somewhat updated and refreshed. We note that if there is no refreshing procedure, then no coding scheme can be secure against continual leakage attack even for one-bit leakage at a times,

\footnotetext{
${ }^{3}$ If there is no refreshing procedure, then the adversary can eventually learn the whole codeword bit-by-bit by leakage. Thus he can learn the underlying message.
} 
so this property is necessary. Our concept of "continuity" is different from that of Faust et al. 23], who considered continual attacks on the same original codeword (the tampering functions can be chosen adaptively). Our model does not allow this type of "resetting attacks." Once a codeword has been modified to $f(C)$, the next tampering function will be applied on $f(C)$.

We remark that the one-time security can be easily extended to the continual case (using a standard hybrid argument) if the update procedure re-encodes the whole underlying messages (c.f. see the results in the work [42]). However, in the setting above, we emphasize on the local property, so this approach does not work. How to do a local update while maintaining tamper and leakage resilience makes the continual case challenging!

Definition 7 (Continual Tampering and Leakage Experiment). Let $k$ be the security parameter, $\mathcal{F}, \mathcal{G}$ be some families of functions. Let (ENC, DEC, UPDATE) be an $(n, \hat{n}, p, q)$-locally decodable and updatable coding scheme with respect to $\Sigma, \hat{\Sigma}$. Let $\mathcal{U}$ be an updater that takes input a message $M \in \Sigma^{n}$ and outputs an index $i \in[n]$ and $m \in \Sigma$. Then for any blocks of messages $M=\left(m_{1}, m_{2}, \ldots, m_{n}\right) \in \Sigma^{n}$, and any (non-uniform) adversary $\mathcal{A}$, any updater $\mathcal{U}$, define the following continual experiment CTamperLeak $_{\mathcal{A}, \mathcal{U}, M}$ :

- The challenger first computes an initial encoding $C^{(1)} \leftarrow \operatorname{ENC}(M)$.

- Then the following procedure repeats, at each round $j$, let $C^{(j)}$ be the current codeword and $M^{(j)}$ be the underlying message:

- $\mathcal{A}$ sends either a tampering function $f \in \mathcal{F}$ and/or a leakage function $g \in \mathcal{G}$ to the challenger.

- The challenger replaces the codeword with $f\left(C^{(j)}\right)$, or sends back a leakage $\ell^{(j)}=g\left(C^{(j)}\right)$.

- We define $\boldsymbol{m}^{(j)} \stackrel{\text { def }}{=}\left(\operatorname{DEC}^{f\left(C^{(j)}\right)}(1), \ldots, \operatorname{DEC}^{f\left(C^{(j)}\right)}(n)\right)$.

- Then the updater computes $\left(i^{(j)}, m\right) \leftarrow \mathcal{U}\left(\boldsymbol{m}^{(j)}\right)$ for the challenger.

- Then the challenger runs UPDATE ${ }^{f\left(C^{(j)}\right)}\left(i^{(j)}, m\right)$ and sends the index $i^{(j)}$ to $\mathcal{A}$.

- $\mathcal{A}$ may terminate the procedure at any point.

- Let $t$ be the total number of rounds above. At the end, the experiment outputs

$$
\left(\ell^{(1)}, \ell^{(2)}, \ldots, \ell^{(t)}, \boldsymbol{m}^{(1)}, \ldots, \boldsymbol{m}^{(t)}, i^{(1)}, \ldots, i^{(t)}\right) .
$$

Definition 8 (Non-malleability and Leakage Resilience against Continual Attacks). An $(n, \hat{n}, p, q)$-locally decodable and updatable coding scheme with respect to $\Sigma, \hat{\Sigma}$ is continual non-malleable against $\mathcal{F}$ and leakage resilient against $\mathcal{G}$ if for all $\mathrm{PPT}$ (non-uniform) adversaries $\mathcal{A}$, and $\mathrm{PPT}$ updaters $\mathcal{U}$, there exists some PPT (non-uniform) simulator $\mathcal{S}$ such that for any $M=\left(m_{1}, \ldots, m_{n}\right) \in$ $\Sigma^{n}$, CTamperLeak CT,U,M $_{\mathcal{A}}$ is (computationally) indistinguishable to the following ideal experiment Ideal $_{\mathcal{S}, \mathcal{U}, M}$ :

- The experiment proceeds in rounds. Let $M^{(1)}=M$ be the initial message.

- At each round $j$, the experiment runs the following procedure: 
- At the beginning of each round, $\mathcal{S}$ outputs $\left(\ell^{(j)}, \mathcal{I}^{(j)}, \boldsymbol{w}^{(j)}\right)$, where $\mathcal{I}^{(j)} \subseteq[n]$.

- Define

$$
\boldsymbol{m}^{(j)}= \begin{cases}\boldsymbol{w}^{(j)} & \text { if } \mathcal{I}^{(j)}=[n] \\ \left.\boldsymbol{m}^{(j)}\right|_{\mathcal{I}^{(j)}}:=\perp,\left.\boldsymbol{m}^{(j)}\right|_{\overline{\mathcal{I}}^{(j)}}:=\left.M^{(j)}\right|_{\overline{\mathcal{I}}^{(j)}} & \text { otherwise }\end{cases}
$$

where $\left.\boldsymbol{x}\right|_{\mathcal{I}}$ denotes the coordinates $\boldsymbol{x}[v]$ where $v \in \mathcal{I}$, and the bar denotes the complement of a set.

- The updater runs $\left(i^{(j)}, m\right) \leftarrow \mathcal{U}\left(\boldsymbol{m}^{(j)}\right)$ and sends the index $i^{(j)}$ to the simulator. Then the experiment updates $M^{(j+1)}$ as follows: set $M^{(j+1)}:=$ $M^{(j)}$ for all coordinates except $i^{(j)}$, and set $M^{(j+1)}\left[i^{(j)}\right]:=m$.

- Let $t$ be the total number of rounds above. At the end, the experiment outputs

$$
\left(\ell^{(1)}, \ell^{(2)}, \ldots, \ell^{(t)}, \boldsymbol{m}^{(1)}, \ldots, \boldsymbol{m}^{(t)}, i^{(1)}, \ldots, i^{(t)}\right) .
$$

\section{Our Constructions}

In this section, we present two constructions. As a warm-up, we first present a construction that is one-time secure to demonstrate the idea of achieving nonmalleability, local decodability and updatability simultaneously. Then in the next section, we show how to make the construction secure against continual attacks. Due to space limit, please find in the full version of the paper [14] the security proofs.

\subsection{A First Attempt - One-time Security}

Construction. Let $\mathcal{E}=$ (Gen, Encrypt, Decrypt) be a symmetric encryption scheme, $\mathrm{NMC}=(\mathrm{ENC}, \mathrm{DEC})$ be a coding scheme. Then we consider the following coding scheme:

- $\operatorname{ENC}(M)$ : on input $M=\left(m_{1}, m_{2}, \ldots, m_{n}\right)$, the algorithm first generates the encryption key sk $\leftarrow \mathcal{E}$.Gen $\left(1^{k}\right)$. Then it computes $c \leftarrow$ NMC.ENC(sk), $e_{i} \leftarrow \mathcal{E}$.Encrypt sk $\left(m_{i}, i\right)$ for $i \in[n]$. The algorithm finally outputs a codeword $C=\left(c, e_{1}, e_{2}, \ldots, e_{n}\right)$.

- $\operatorname{DEC}^{C}(i)$ : on input $i \in[n]$, the algorithm reads the first block and the $(i+1)$-st block of the codeword to retrieve $\left(c, e_{i}\right)$. Then it runs sk $=N$ NMC.DEC $(c)$. If the decoding algorithm outputs $\perp$, then it outputs $\perp$ and terminates. Else, it computes $\left(m_{i}, i^{*}\right)=\mathcal{E}$.Decrypt sk $\left(e_{i}\right)$. If $i^{*} \neq i$, or the decryption fails, the algorithm outputs $\perp$. If all the above verifications pass, the algorithm outputs $m_{i}$.

- $\operatorname{update}\left(i, m^{\prime}\right):$ on inputs an index $i \in[n]$, a block of message $m^{\prime} \in \Sigma$, the algorithm runs $\operatorname{DEC}^{C}(i)$ to retrieve $\left(c, e_{i}\right)$ and $\left(\mathrm{sk}, m_{i}, i\right)$. If the decoding algorithm returns $\perp$, the algorithm writes $\perp$ to the first block and the $(i+1)$ st block. Otherwise, it computes a fresh encoding $c^{\prime} \leftarrow$ NMC.ENC(sk), and a fresh ciphertext $e_{i}^{\prime} \leftarrow \mathcal{E}$. Encrypt sk $\left(m^{\prime}, i\right)$. Then it writes back the first block and the $(i+1)$-st block with $\left(c^{\prime}, e_{i}^{\prime}\right)$. 
To analyze the coding scheme, we make the following assumptions of the parameters in the underlying scheme for convenience:

1. The size of the encryption key is $k$ (security parameter), i.e. $|\mathrm{sk}|=k$.

2. Let $\Sigma$ be a set, and the encryption scheme supports messages of length $|\Sigma|+\log n$. The ciphertexts are in the space $\hat{\Sigma}$.

3. The length of $|\mathrm{NMC.ENC}(\mathrm{sk})|$ is less than $|\hat{\Sigma}|$.

Then clearly, the above coding scheme is an $(n, n+1,2,2)$-locally updatable and decodable code with respect to $\Sigma, \hat{\Sigma}$. The correctness of the scheme is obvious by inspection. The rate (ratio of the length of messages to that of codewords) of the coding scheme is $1-o(1)$.

Theorem 1. Assume $\mathcal{E}$ is a symmetric authenticated encryption scheme, and NMC is a non-malleable code against the tampering function class $\mathcal{F}$. Then the coding scheme presented above is one-time non-malleable against the tampering class

$$
\overline{\mathcal{F}} \stackrel{\text { def }}{=}\left\{\begin{array}{l}
f: \hat{\Sigma}^{n+1} \rightarrow \hat{\Sigma}^{n+1} \text { and }|f| \leq \operatorname{poly}(k), \text { such that }: \\
f=\left(f_{1}, f_{2}\right), f_{1}: \hat{\Sigma}^{n+1} \rightarrow \hat{\Sigma}, f_{2}: \hat{\Sigma}^{n} \rightarrow \hat{\Sigma}^{n} \\
\forall\left(x_{2}, \ldots, x_{n+1}\right) \in \hat{\Sigma}^{n}, f_{1}\left(\cdot, x_{2}, \ldots, x_{n+1}\right) \in \mathcal{F} \\
f\left(x_{1}, x_{2}, \ldots, x_{n+1}\right)=\left(f_{1}\left(x_{1}, x_{2}, \ldots, x_{n+1}\right), f_{2}\left(x_{2}, \ldots, x_{n+1}\right)\right) .
\end{array}\right\} .
$$

We have presented the intuition in the introduction. Before giving the detailed proof, we make the following remark.

Remark 4. The function class $\overline{\mathcal{F}}$ may look complex, yet the intuition is simple. The tampering function restricted in the first block (the underlying nonmalleable code) falls into the class $\mathcal{F}$ - this is captured by $f_{1} \in \mathcal{F}$; on the other hand, we just require the function restricted in the rest of the blocks to be polynomial-sized - this is captured by $\left|f_{2}\right| \leq|f| \leq \operatorname{poly}(k)$.

For our construction, it is inherent that the function $f_{2}$ cannot depend on $x_{1}$ arbitrarily. Suppose this is not the case, then $f_{2}$ can first decode the nonmalleable code, encrypt the decoded value and write the ciphertext into $x_{2}$, which breaks non-malleability. However, if the underlying coding scheme is nonmalleable and also leakage resilient to $\mathcal{G}$, then we can allow $f_{2}$ to get additional information $g\left(x_{1}\right)$ for any $g \in \mathcal{G}$. Moreover, the above construction is one-time leakage resilient.

We present the above simpler version for clarity of exposition, and give this remark that our construction actually achieves security against a broader class of tampering attacks.

\subsection{Achieving Security against Continual Attacks}

As discussed in the introduction, the above construction is not secure if continual tampering and leakage is allowed - the adversary can use a rewind attack to modify the underlying message to some old/related messages. We handle this challenge using a technique of Merkle tree, which preserves local properties of the above scheme. We present the construction in the following: 
Definition 9 (Merkle Tree). Let $h: \mathcal{X} \times \mathcal{X} \rightarrow \mathcal{X}$ be a hash function that maps two blocks of messages to one 4 A Merkle Tree $\operatorname{Tree}_{h}(M)$ takes input a message $M=\left(m_{1}, m_{2}, \ldots, m_{n}\right) \in \mathcal{X}^{n}$. Then it applies the hash on each pair $\left(m_{2 i-1}, m_{2 i}\right)$, and resulting in $n / 2$ blocks. Then again, it partitions the blocks into pairs and applies the hash on the pairs, which results in $n / 4$ blocks. This is repeated $\log n$ times, resulting a binary tree with hash values, until one block remains. We call this value the root of Merkle Tree denoted $\operatorname{Root}_{h}(M)$, and the internal nodes (including the root) as $\operatorname{Tree}_{h}(M)$. Here $M$ can be viewed as leaves.

Theorem 2. Assuming $h$ is a collision resistant hash function. Then for any message $M=\left(m_{1}, m_{2}, \ldots, m_{n}\right) \in \mathcal{X}^{n}$ and any polynomial time adversary $\mathcal{A}$, $\operatorname{Pr}\left[\left(m_{i}^{\prime}, p_{i}\right) \leftarrow \mathcal{A}(M, h): m_{i}^{\prime} \neq m_{i}, p_{i}\right.$ is a consistent path with $\left.\operatorname{Root}_{h}(M)\right] \leq$ $\operatorname{negl}(k)$.

Moreover, given a path $p_{i}$ passing the leaf $m_{i}$, and a new value $m_{i}^{\prime}$, there is an algorithm that computes $\operatorname{Root}_{h}\left(M^{\prime}\right)$ in time poly $(\log n, k)$, where $M^{\prime}=$ $\left(m_{1}, \ldots, m_{i-1}, m_{i}^{\prime}, m_{i+1}, \ldots, m_{n}\right)$.

Construction. Let $\mathcal{E}=$ (Gen, Encrypt, Decrypt) be a symmetric encryption scheme, $\mathrm{NMC}=(\mathrm{ENC}, \mathrm{DEC})$ be a non-malleable code, $H$ is a family of collision resistance hash functions. Then we consider the following coding scheme:

- $\operatorname{ENC}(M)$ : on input $M=\left(m_{1}, m_{2}, \ldots, m_{n}\right)$, the algorithm first generates encryption key sk $\leftarrow \mathcal{E}$.Gen $\left(1^{k}\right)$ and $h \leftarrow H$. Then it computes $e_{i} \leftarrow$ $\mathcal{E}$.Encrypt sk $\left(m_{i}\right)$ for $i \in[n]$, and $T=\operatorname{Tree}_{h}\left(e_{1}, \ldots, e_{n}\right), R=\operatorname{Root}_{h}\left(e_{1}, \ldots, e_{n}\right)$. Then it computes $c \leftarrow$ NMC.ENC(sk, $R, h)$, The algorithm finally outputs a codeword $C=\left(c, e_{1}, e_{2}, \ldots, e_{n}, T\right)$.

- $\operatorname{DEC}^{C}(i)$ : on input $i \in[n]$, the algorithm reads the first block, the $(i+1)$-st block, and a path $p$ in the tree (from the root to the leaf $i$ ), and it retrieve $\left(c, e_{i}, p\right)$. Then it runs $(\mathrm{sk}, R, h)=\mathrm{NMC} \cdot \operatorname{DEC}(c)$. If the decoding algorithm outputs $\perp$, or the path is not consistent with the root $R$, then it outputs $\perp$ and terminates. Else, it computes $m_{i}=\mathcal{E}$.Decrypt sk $\left(e_{i}\right)$. If the decryption fails, output $\perp$. If all the above verifications pass, the algorithm outputs $m_{i}$.

- $\operatorname{Update}\left(i, m^{\prime}\right)$ : on inputs an index $i \in[n]$, a block of message $m^{\prime} \in \Sigma$, the algorithm runs $\mathrm{DEC}^{C}(i)$ to retrieve $\left(c, e_{i}, p\right)$. Then the algorithm can derive $(\mathrm{sk}, R, h)=\mathrm{NMC}$. $\operatorname{DEC}(c)$. If the decoding algorithm returns $\perp$, the update writes $\perp$ to the first block, which denotes failure. Otherwise, it computes a fresh ciphertext $e_{i}^{\prime} \leftarrow \mathcal{E}$. Encrypt sk $\left(m^{\prime}\right)$, a new path $p^{\prime}$ (that replaces $e_{i}$ by $e_{i}^{\prime}$ ) and a new root $R^{\prime}$, which is consistent with the new leaf value $e_{i}^{\prime}$. (Note that this can be done given only the old path $p$ as Theorem 2, Finally, it computes a fresh encoding $c^{\prime} \leftarrow$ NMC.ENC $\left(\mathrm{sk}, R^{\prime}, h\right)$. Then it writes back the first block, the $(i+1)$-st block, and the new path blocks with $\left(c^{\prime}, e_{i}^{\prime}, p^{\prime}\right)$.

To analyze the coding scheme, we make the following assumptions of the parameters in the underlying scheme for convenience:

1. The size of the encryption key is $k$ (security parameter), i.e. $|\mathbf{s k}|=k$ and the length of the output of the hash function is $k$.

\footnotetext{
${ }^{4}$ Here we assume $|\mathcal{X}|$ is greater than the security parameter.
} 
2. Let $\Sigma$ be a set, and the encryption scheme supports messages of length $|\Sigma|$. The ciphertexts are in the space $\hat{\Sigma}$.

3. The length of $|\operatorname{NMC.ENC}(\mathbf{s k}, v)|$ is less than $|\hat{\Sigma}|$, where $|v|=k$.

Clearly, the above coding scheme is an $(n, 2 n+1, O(\log n), O(\log n))$-locally updatable and decodable code with respect to $\Sigma, \hat{\Sigma}$. The correctness of the scheme is obvious by inspection. The rate (ratio of the length of messages to that of codewords) of the coding scheme is $1 / 2-o(1)$.

Theorem 3. Assume $\mathcal{E}$ is a semantically secure symmetric encryption scheme, and NMC is a non-malleable code against the tampering function class $\mathcal{F}$, and leakage resilient against the function class $\mathcal{G}$. Then the coding scheme presented above is non-malleable against continual attacks of the tampering class

$$
\overline{\mathcal{F}} \stackrel{\text { def }}{=}\left\{\begin{array}{l}
f: \hat{\Sigma}^{2 n+1} \rightarrow \hat{\Sigma}^{2 n+1} \text { and }|f| \leq \operatorname{poly}(k), \text { such that : } \\
f=\left(f_{1}, f_{2}\right), f_{1}: \hat{\Sigma}^{2 n+1} \rightarrow \hat{\Sigma}, f_{2}: \hat{\Sigma}^{2 n} \rightarrow \hat{\Sigma}^{2 n} \\
\forall\left(x_{2}, \ldots, x_{2 n+1}\right) \in \hat{\Sigma}^{n}, f_{1}\left(\cdot, x_{2}, \ldots, x_{2 n+1}\right) \in \mathcal{F}, \\
f\left(x_{1}, x_{2}, \ldots, x_{2 n+1}\right)=\left(f_{1}\left(x_{1}, x_{2}, \ldots, x_{2 n+1}\right), f_{2}\left(x_{2}, \ldots, x_{2 n+1}\right)\right) .
\end{array}\right\},
$$

and is leakage resilient against the class

$$
\overline{\mathcal{G}} \stackrel{\text { def }}{=}\left\{\begin{array}{l}
g: \hat{\Sigma}^{2 n+1} \rightarrow \mathcal{Y} \text { and }|g| \leq \text { poly }(k), \text { such that }: \\
g=\left(g_{1}, g_{2}\right), g_{1}: \hat{\Sigma}^{2 n+1} \rightarrow \mathcal{Y}^{\prime}, g_{2}: \hat{\Sigma}^{2 n} \rightarrow \hat{\Sigma}^{2 n}, \\
\forall\left(x_{2}, \ldots, x_{2 n+1}\right) \in \hat{\Sigma}^{n}, g_{1}\left(\cdot, x_{2}, \ldots, x_{2 n+1}\right) \in \mathcal{G} .
\end{array}\right\} .
$$

The intuition of this construction can be found in the introduction. Before giving the detailed proof, we make a remark.

Remark 5. Actually our construction is secure against a broader class of tampering functions. The $f_{2}$ part can depend on $g^{\prime}\left(x_{1}\right)$ as long as the function $g^{\prime}(\cdot)$ together with the leakage function $g_{1}\left(\cdot, x_{2}, \ldots, x_{2 n+1}\right)$ belong to $\mathcal{G}$. That is, the tampering function $f=\left(f_{1}, f_{2}, g^{\prime}\right)$ and the leakage function $g=\left(g_{1}, g_{2}\right)$ satisfy the constraint $g^{\prime}(\cdot) \circ g_{1}\left(\cdot, x_{2}, \ldots, x_{2 n+1}\right) \in \mathcal{G}$ (Here we use $\circ$ to denote concatenation). For presentation clarity, we choose to describe the simpler but slightly smaller class of functions.

\subsection{Instantiations}

In this section, we describe several constructions of non-malleable codes against different classes of tampering/leakage functions. To our knowledge, we can use the explicit constructions (of the non-malleable codes) in the work [1, 3, $, 2,22,23$, 25. 42 .

First we overview different classes of tampering/leakage function allowed for these results: the constructions of 22 work for bit-wise tampering functions, and split-state functions in the random oracle model. The construction of Choi et al. 9] works for small block tampering functions. The construction of Liu and Lysyanskaya 42 achieves both tamper and leakage resilience against splitstate functions in the common reference string (CRS) model. The construction of 
Dziembowski et al. [20] achieves information theoretic security against split-state tampering functions, but their scheme can only support encoding for bits, so it cannot be used in our construction. The subsequent construction by Aggarwal et al. 1] achieves information theoretic security against split-state tampering without CRS. We believe that their construction also achieves leakage resilience against some length bounded split-state leakage yet their paper did not claim it. The construction by Faust et al. 25. is non-malleable against small-sized tampering functions. Another construction by Faust et al. 23] achieves both tamper and leakage resilience in the split-state model with CRS. The construction of Aggarwal et al. [3] is non-malleable against permutation functions.

Then we remark that actually there are other non-explicit constructions: Cheraghchi and Guruswami [8] showed the relation non-malleable codes and nonmalleable two source extractors (but constructing a non-malleable two-source extractor is still open), and in another work Cheraghchi and Guruswami [7] showed the existence of high rate non-malleable codes in the split-state model but did not give an explicit (efficient) construction.

Finally, we give a concrete example of what the resulting class looks like using the construction of Liu and Lysyanskaya [42] as building block. Recall that their construction achieves both tamper and leakage resilience for split-state functions. Our construction has the form:

$$
\text { (NMC.ENC(sk, } \left.h, T), \operatorname{Encrypt}\left(m_{1}\right), \ldots, \operatorname{Encrypt}\left(m_{n}\right), T\right)
$$

So the overall leakage function $g$ restricted in the first block (i.e. $g_{1}$ ) can be a (poly-sized) length-bounded split-state function; $g$ on the other hand, can leak all the other parts. For the tampering, the overall tampering function $f$ restricted in the first block (i.e. $f_{1}$ ) can be any (poly-sized) split-state function. On the other hand $f$ restricted in the rest (i.e. $f_{2}$ ) can be just any poly-sized function. We also remark that $f_{2}$ can depend on a split-state leakage on the first part, say $g_{1}$, as we discussed in the previous remark above.

\section{Tamper and Leakage Resilient RAM}

In this section, we first introduce the notations of the Random Access Machine (RAM) model of computation in presence of tampering and leakage attacks in Section 4.1. Then we define the security of tamper and leakage resilient RAM model of computation in Section 4.2, and then give a construction in Section 4.3 . Due to space limit, please find in the full version of the paper [14, the building block Oblivious RAM (ORAM), more detailed construction, and the security analysis.

\subsection{Random Access Machines}

We consider RAM programs to be interactive stateful systems $\langle\Pi$, state, $D\rangle$, where $\Pi$ denotes a next instruction function, state the current state stored in registers, and $D$ the content of memory. Upon state and an input value $d$, the next instruction function outputs the next instruction $I$ and an updated state state'. 
The initial state of the RAM machine, state, is set to (start,*). For simplicity we often denote RAM program as $\langle\Pi, D\rangle$. We consider four ways of interacting with the system:

- Execute $(x)$ : A user can provide the system with Execute $(x)$ queries, for $x \in$ $\{0,1\}^{u}$, where $u$ is the input length. Upon receiving such query, the system computes $\left(y, t, D^{\prime}\right) \leftarrow\langle\Pi, D\rangle(x)$, updates the state of the system to $D:=D^{\prime}$ and outputs $(y, t)$, where $y$ denotes the output of the computation and $t$ denotes the time (or number of executed instructions). By Execute $_{1}(x)$ we denote the first coordinate of the output of Execute $(x)$.

- $\operatorname{doNext}(x)$ : A user can provide the system with doNext $(x)$ queries, for $x \in$ $\{0,1\}^{u}$. Upon receiving such query, if state $=($ start, $*)$, set state $:=($ start,$x)$, and $d:=0^{r}$; Here $\rho=\mid$ state $\mid$ and $r=|d|$. The system does the following until termination:

1. Compute $\left(I\right.$, state $\left.^{\prime}\right)=\Pi($ state,$d)$. Set state $:=$ state $^{\prime}$.

2 . If $I=$ (wait) then set state $:=0^{\rho}, d:=0^{r}$ and terminate.

3. If $I=($ stop, $z)$ then set state $:=($ start, $*), d:=0^{r}$ and terminate with output $z$.

4. If $I=\left(\right.$ write, $\left.v, d^{\prime}\right)$ then set $D[v]:=d^{\prime}$.

5 . If $I=(\mathrm{read}, v, \perp)$ then set $d:=D[v]$.

Let $I_{1}, \ldots, I_{\ell}$ be the instructions executed by doNext $(x)$. All memory addresses of executed instructions are returned to the user. Specifically, for instructions $I_{j}$ of the form (read, $v, \perp$ ) or (write, $v, d^{\prime}$ ), $v$ is returned.

- Tamper $(f)$ : We also consider tampering attacks against the system, modeled by Tamper $(f)$ commands, for functions $f$. Upon receiving such command, the system sets $D:=f(D)$.

- Leak $(g)$ : We finally consider leakage attacks against the system, modeled by Leak $(g)$ commands, for functions $g$. Upon receiving such command, the value of $g(D)$ is returned to the user.

Remark 6. A doNext $(x)$ instruction groups together instructions performed by the $\mathrm{CPU}$ in a single clock cycle. Intuitively, a (wait) instruction indicates that a clock cycle has ended and the CPU waits for the adversary to increment the clock. In contrast, a (stop, $z$ ) instruction indicates that the entire execution has concluded with output $z$. In this case, the internal state is set back to the start state.

We require that each $\operatorname{doNext}(x)$ operation performs exactly $\ell=\ell(k)=\operatorname{poly}(k)$ instructions $I_{1}, \ldots, I_{\ell}$ where: The final instruction is of the form $I_{\ell}=($ stop, $\cdot)$ or $I_{\ell}=$ (wait). For fixed $\ell_{1}=\ell_{1}(k), \ell_{2}=\ell_{2}(k)$ such that $\ell_{1}+\ell_{2}=\ell-1$, we have that the first $\ell_{1}$ instructions are of the form $I_{\ell}=(\mathrm{read}, \cdot, \perp)$ and the next $\ell_{2}$ instructions are of the form $I_{\ell}=$ (write, $v, d^{\prime}$ ). We assume that $\ell, \ell_{1}, \ell_{2}$ are implementation-specific and public. The limitations on space are meant to model the fact that the CPU has a limited number of registers and that no persistent state is kept by the CPU between clock cycles.

Remark \%. We note that Execute $(x)$ instructions are used by the ideal world adversary - who learns only the input-output behavior of the RAM machine and 
the run time - as well as by the real world adversary. The real world adversary may also use the more fine-grained doNext $(x)$ instruction. We note that given access to the doNext $(x)$ instruction, the behavior of the Execute $(x)$ instruction may be simulated.

Remark 8. We note that our model does not explicitly allow for leakage and tampering on instructions $I$. E.g. when an instruction $I=$ (write, $v, d^{\prime}$ ) is executed, we do not directly allow tampering with the values $v, d^{\prime}$ or leakage on $d^{\prime}$ (note that $v$ is entirely leaked to the adversary). Nevertheless, as discussed in the introduction, since we allow full leakage on the addresses, the adversary can use the tampering and leakage attacks on the memory to capture the attacks on the instructions. We defer a rigorous treatment and analysis of such attacks to future work. In this work, for simplicity of presentation we assume these instructions are not subject to direct attacks.

\subsection{Tamper and Leakage-Resilient (TLR) RAM}

A tamper and leakage resilient (TLR) RAM compiler consists of two algorithms (CompMem, CompNext), which transform a RAM program $\langle\Pi, D\rangle$ into another program $\langle\widehat{\Pi}, \widehat{D}\rangle$ as follows: On input database $D$, CompMem initializes the memory and internal state of the compiled machine, and generates the transformed database $\widehat{D}$; On input next instruction function $\Pi$, CompNext generates the next instruction function of the compiled machine.

Definition 10. A TLR compiler (CompMem, CompNext) is tamper and leakage simulatable w.r.t. function families $\mathcal{F}, \mathcal{G}$, if for every $R A M$ next instruction function $\Pi$, and for any $\mathrm{PPT}$ (non-uniform) adversary $\mathcal{A}$ there exists a $\mathrm{PPT}$ (non-uniform) simulator $\mathcal{S}$ such that for any initial database $D \in\{0,1\}$ poly $(k)$ we have

$\operatorname{TamperExec}(\mathcal{A}, \mathcal{F}, \mathcal{G},\langle\operatorname{CompNext}(\Pi), \operatorname{CompMem}(D)\rangle) \approx \operatorname{IdealExec}(\mathcal{S},\langle\Pi, D\rangle)$

where TamperExec and IdealExec are defined as follows:

- $\operatorname{TamperExec}(\mathcal{A}, \mathcal{F}, \mathcal{G},\langle\operatorname{CompNext}(\Pi), \operatorname{CompMem}(D)\rangle):$ The adversary $\mathcal{A}$ interacts with the system $\langle\operatorname{CompNext}(\Pi)$, CompMem $(D)\rangle$ for arbitrarily many rounds of interactions where, in each round:

1. The adversary can "tamper" by executing a Tamper $(f)$ command against the system, for some $f \in \mathcal{F}$.

2. The adversary can "leak" by executing a Leak $(g)$ command against the system, and receiving $g(D)$ in return.

3. The adversary requests a doNext $(x)$ command to be executed by the system. Let $I_{1}, \ldots, I_{\ell}$ be the instructions executed by $\operatorname{doNext}(x)$. If $I_{\ell}$ is of the form (stop, $z$ ) then output $z$ is returned to the adversary. Moreover, all memory addresses corresponding to instructions $I_{1}, \ldots, I_{\ell-1}$ are returned to the adversary. 
The output of the game consists of the output of the adversary $\mathcal{A}$ at the end of the interaction, along with (1) all input-output pairs $\left(x_{1}, y_{1}\right),\left(x_{2}, y_{2}\right), \ldots$, (2) all responses to leakage queries $\ell_{1}, \ell_{2}, \ldots$ (3) all outputs of $\operatorname{doN} \operatorname{Next}\left(x_{1}\right)$, $\operatorname{doNext}\left(x_{2}\right), \ldots$

- IdealExec $(\mathcal{S},\langle\Pi, D\rangle):$ The simulator interacts with the system $\langle\Pi, D\rangle$ for arbitrarily many rounds of interaction where, in each round, it runs an Execute $(x)$ query for some $x \in\{0,1\}^{u}$ and receives output $(y, t)$. The output of the game consists of the output of the simulator $\mathcal{S}$ at the end of the interaction, along with all of the execute-query inputs and outputs.

For simplicity of exposition, we assume henceforth that the next instruction function $\Pi$ to be compiled is the universal RAM next instruction function. In other words, we assume that the program to be executed is stored in the initial database $D$.

\subsection{TLR-RAM Construction}

Here we first give a high-level description of our construction and then state our theorem. More detailed construction and the security proof will be given in the full version of the paper 14 .

High-level Description of Construction Let $D$ be the initial database and let ORAM $=$ (oCompMem, oCompNext) be an ORAM compiler. Let NMCode = (ENC, DEC, UPDATE) be a locally decodable and updatable code. We present the following construction TLR-RAM = (CompMem, CompNext) of a tamper and leakage resilient RAM compiler. In order to make our presentation more intuitive, instead of specifying the next message function CompNext $(\Pi)$, we specify the pseudocode for the $\operatorname{doNext}(x)$ instruction of the compiled machine. We note that CompNext $(\Pi)$ is implicitly defined by this description.

TLR-RAM takes as input an initial database $D$ and a next instruction function $\Pi$ and does the following:

- CompMem: On input security parameter $k$ and initial database $D$, CompMem does:

- Compute $\widetilde{D} \leftarrow$ oCompMem $(D)$, and output $\widehat{D} \leftarrow \operatorname{ENC}(\widetilde{D})$.

- Initialize the ORAM state stateorAm $:=($ start, $*)$ and $d_{\text {ORAM }}:=0^{r}$, where $r=\left|d_{\text {ORAM }}\right|$.

- $\operatorname{doNext}(x)$ : On input $x$, do the following until termination:

1. If $d_{\text {ORAM }}=\perp$ then abort.

2. Compute $\left(I\right.$, state ORAM $\left.^{\prime}\right) \leftarrow$ oCompNext $(\Pi)$ (state oram,$\left.d_{\text {ORAM }}\right)$ and set state ORAM $:=$ state $_{\text {ORAM }}$.

3. If $I=$ (wait) then set stateorAM $:=0^{\rho}$ and $d_{\mathrm{ORAM}}:=0^{r}$ and terminate. Here $\rho=\mid$ stateoram $\mid$ and $r=\left|d_{\text {ORAM }}\right|$.

4. If $I=($ stop, $z)$ then set stateoram $:=($ start, $*), d:=0^{r}$ and terminate with output $z$.

5. If $I=\left(\right.$ write, $\left.v, d^{\prime}\right)$ then run $\operatorname{UPDATE}^{\widehat{D}}\left(v, d^{\prime}\right)$. 
6. If $I=(\operatorname{read}, v, \perp)$ then set $d_{\text {ORAM }}:=\operatorname{DEC}^{\widehat{D}}(v)$.

We are now ready to present the main theorem of this section:

Theorem 4. Assume ORAM = (oCompMem, oCompNext) is an ORAM compiler which is access-pattern hiding and assume NMCode = (ENC, DEC, UPDATE) is a locally decodable and updatable code which is continual non-malleable against $\mathcal{F}$ and leakage resilient against $\mathcal{G}$. Then TLR-RAM $=$ (CompMem, CompNext) presented above is tamper and leakage simulatable w.r.t. function families $\mathcal{F}, \mathcal{G}$.

Acknowledgement. We thank Yevgeniy Dodis for helpful discussions. This research was funded in part by an NSF grant CNS-1314857, a subcontract from the DARPA PROCEED program, a Sloan Research Fellowship, and Google Faculty Research Awards. The views and conclusions contained herein are those of the authors and should not be interpreted as representing funding agencies.

\section{References}

1. Aggarwal, D., Dodis, Y., Lovett, S.: Non-malleable codes from additive combinatorics. In: STOC (2014), http://eprint.iacr.org/2013/201

2. Agrawal, D., Archambeault, B., Rao, J.R., Rohatgi, P.: The EM side-channel(s). In: Kaliski Jr., B.S., Koç, Ç.K., Paar, C. (eds.) CHES 2002. LNCS, vol. 2523, pp. 29-45. Springer, Heidelberg (2003)

3. Agrawal, S., Gupta, D., Maji, H.K., Pandey, O., Prabhakaran, M.: Explicit nonmalleable codes resistant to permutations. In: Cryptology ePrint Archive, Report 2014/316 (2014)

4. Biham, E., Shamir, A.: Differential fault analysis of secret key cryptosystems. In: Kaliski Jr., B.S. (ed.) CRYPTO 1997. LNCS, vol. 1294, pp. 513-525. Springer, Heidelberg (1997)

5. Boneh, D., DeMillo, R.A., Lipton, R.J.: On the importance of eliminating errors in cryptographic computations. Journal of Cryptology 14(2), 101-119 (2001)

6. Chandran, N., Kanukurthi, B., Ostrovsky, R.: Locally updatable and locally decodable codes. In: Lindell, Y. (ed.) TCC 2014. LNCS, vol. 8349, pp. 489-514. Springer, Heidelberg (2014)

7. Cheraghchi, M., Guruswami, V.: Capacity of non-malleable codes. In: Naor, M. (ed.) ITCS 2014, pp. 155-168. ACM (January 2014)

8. Cheraghchi, M., Guruswami, V.: Non-malleable coding against bit-wise and splitstate tampering. In: Lindell, Y. (ed.) TCC 2014. LNCS, vol. 8349, pp. 440-464. Springer, Heidelberg (2014)

9. Choi, S.G., Kiayias, A., Malkin, T.: BiTR: Built-in tamper resilience. In: Lee, D.H., Wang, X. (eds.) ASIACRYPT 2011. LNCS, vol. 7073, pp. 740-758. Springer, Heidelberg (2011)

10. Chor, B., Kushilevitz, E., Goldreich, O., Sudan, M.: Private information retrieval. J. ACM 45(6), 965-981 (1998)

11. Coretti, S., Maurer, U., Tackmann, B., Venturi, D.: From single-bit to multi-bit public-key encryption via non-malleable codes. In: Cryptology ePrint Archive, Report 2014/324 (2014) 
12. Dachman-Soled, D., Kalai, Y.T.: Securing circuits against constant-rate tampering. In: Safavi-Naini, R., Canetti, R. (eds.) CRYPTO 2012. LNCS, vol. 7417, pp. 533-551. Springer, Heidelberg (2012)

13. Dachman-Soled, D., Kalai, Y.T.: Securing circuits and protocols against $1 / \operatorname{poly}(k)$ tampering rate. In: Lindell, Y. (ed.) TCC 2014. LNCS, vol. 8349, pp. 540-565. Springer, Heidelberg (2014)

14. Dachman-Soled, D., Liu, F.-H., Shi, E., Zhou, H.-S.: Locally decodable and updatable non-malleable codes and their applications. Cryptology ePrint Archive, Report 2014/663 (2014), http://eprint.iacr.org/2014/663

15. Damgård, I., Faust, S., Mukherjee, P., Venturi, D.: Bounded tamper resilience: How to go beyond the algebraic barrier. In: Sako, K., Sarkar, P. (eds.) ASIACRYPT 2013, Part II. LNCS, vol. 8270, pp. 140-160. Springer, Heidelberg (2013)

16. Dodis, Y., Pietrzak, K.: Leakage-resilient pseudorandom functions and side-channel attacks on Feistel networks. In: Rabin, T. (ed.) CRYPTO 2010. LNCS, vol. 6223, pp. 21-40. Springer, Heidelberg (2010)

17. Duc, A., Dziembowski, S., Faust, S.: Unifying leakage models: From probing attacks to noisy leakage. In: Nguyen, P.Q., Oswald, E. (eds.) EUROCRYPT 2014. LNCS, vol. 8441, pp. 423-440. Springer, Heidelberg (2014)

18. Dziembowski, S., Faust, S.: Leakage-resilient cryptography from the inner-product extractor. In: Lee, D.H., Wang, X. (eds.) ASIACRYPT 2011. LNCS, vol. 7073, pp. 702-721. Springer, Heidelberg (2011)

19. Dziembowski, S., Faust, S.: Leakage-resilient circuits without computational assumptions. In: Cramer, R. (ed.) TCC 2012. LNCS, vol. 7194, pp. 230-247. Springer, Heidelberg (2012)

20. Dziembowski, S., Kazana, T., Obremski, M.: Non-malleable codes from two-source extractors. In: Canetti, R., Garay, J.A. (eds.) CRYPTO 2013, Part II. LNCS, vol. 8043, pp. 239-257. Springer, Heidelberg (2013)

21. Dziembowski, S., Pietrzak, K.: Leakage-resilient cryptography. In: 49th FOCS, pp. 293-302. IEEE Computer Society Press (October 2008)

22. Dziembowski, S., Pietrzak, K., Wichs, D.: Non-malleable codes. In: Yao, A.C.-C. (ed.) ICS 2010, pp. 434-452. Tsinghua University Press (January 2010)

23. Faust, S., Mukherjee, P., Nielsen, J.B., Venturi, D.: Continuous non-malleable codes. In: Lindell, Y. (ed.) TCC 2014. LNCS, vol. 8349, pp. 465-488. Springer, Heidelberg (2014)

24. Faust, S., Mukherjee, P., Nielsen, J.B., Venturi, D.: A tamper and leakage resilient random access machine. In: Cryptology ePrint Archive, Report 2014/338 (2014)

25. Faust, S., Mukherjee, P., Venturi, D., Wichs, D.: Efficient non-malleable codes and key-derivation for poly-size tampering circuits. In: Nguyen, P.Q., Oswald, E. (eds.) EUROCRYPT 2014. LNCS, vol. 8441, pp. 111-128. Springer, Heidelberg (2014)

26. Faust, S., Pietrzak, K., Venturi, D.: Tamper-proof circuits: How to trade leakage for tamper-resilience. In: Aceto, L., Henzinger, M., Sgall, J. (eds.) ICALP 2011, Part I. LNCS, vol. 6755, pp. 391-402. Springer, Heidelberg (2011)

27. Faust, S., Rabin, T., Reyzin, L., Tromer, E., Vaikuntanathan, V.: Protecting circuits from leakage: the computationally-bounded and noisy cases. In: Gilbert, H. (ed.) EUROCRYPT 2010. LNCS, vol. 6110, pp. 135-156. Springer, Heidelberg (2010)

28. Gennaro, R., Lysyanskaya, A., Malkin, T., Micali, S., Rabin, T.: Algorithmic tamper-proof (ATP) security: Theoretical foundations for security against hardware tampering. In: Naor, M. (ed.) TCC 2004. LNCS, vol. 2951, pp. 258-277. Springer, Heidelberg (2004) 
29. Goldreich, O., Ostrovsky, R.: Software protection and simulation on oblivious rams. Journal of the ACM 43(3), 431-473 (1996)

30. Goldwasser, S., Rothblum, G.N.: Securing computation against continuous leakage. In: Rabin, T. (ed.) CRYPTO 2010. LNCS, vol. 6223, pp. 59-79. Springer, Heidelberg (2010)

31. Goldwasser, S., Rothblum, G.N.: How to compute in the presence of leakage. In: 53rd FOCS, pp. 31-40. IEEE Computer Society Press (October 2012)

32. Halderman, J.A., Schoen, S.D., Heninger, N., Clarkson, W., Paul, W., Calandrino, J.A., Feldman, A.J., Appelbaum, J., Felten, E.W.: Lest we remember: Cold boot attacks on encryption keys. In: USENIX Security Symposium, pp. 45-60 (2008)

33. Ishai, Y., Kushilevitz, E.: On the hardness of information-theoretic multiparty computation. In: Cachin, C., Camenisch, J.L. (eds.) EUROCRYPT 2004. LNCS, vol. 3027, pp. 439-455. Springer, Heidelberg (2004)

34. Ishai, Y., Prabhakaran, M., Sahai, A., Wagner, D.: Private circuits II: Keeping secrets in tamperable circuits. In: Vaudenay, S. (ed.) EUROCRYPT 2006. LNCS, vol. 4004, pp. 308-327. Springer, Heidelberg (2006)

35. Ishai, Y., Sahai, A., Wagner, D.: Private circuits: Securing hardware against probing attacks. In: Boneh, D. (ed.) CRYPTO 2003. LNCS, vol. 2729, pp. 463-481. Springer, Heidelberg (2003)

36. Juma, A., Vahlis, Y.: Protecting cryptographic keys against continual leakage. In: Rabin, T. (ed.) CRYPTO 2010. LNCS, vol. 6223, pp. 41-58. Springer, Heidelberg (2010)

37. Katz, J., Trevisan, L.: On the efficiency of local decoding procedures for errorcorrecting codes. In: 32nd ACM STOC, pp. 80-86. ACM Press (May 2000)

38. Kiayias, A., Tselekounis, Y.: Tamper resilient circuits: The adversary at the gates. In: Sako, K., Sarkar, P. (eds.) ASIACRYPT 2013, Part II. LNCS, vol. 8270, pp. 161-180. Springer, Heidelberg (2013)

39. Paul, C.: Timing attacks on implementations of Diffie-Hellman, RSA, DSS, and other systems. In: Koblitz, N. (ed.) CRYPTO 1996. LNCS, vol. 1109, pp. 104-113. Springer, Heidelberg (1996)

40. Kocher, P., Jaffe, J., Jun, B.: Differential power analysis. In: Wiener, M. (ed.) CRYPTO 1999. LNCS, vol. 1666, pp. 388-397. Springer, Heidelberg (1999)

41. Lie, D., Thekkath, C.A., Mitchell, M., Lincoln, P., Boneh, D., Mitchell, J.C., Horowitz, M.: Architectural support for copy and tamper resistant software. In: ASPLOS, pp. 168-177 (2000)

42. Liu, F.-H., Lysyanskaya, A.: Tamper and leakage resilience in the split-state model. In: Safavi-Naini, R., Canetti, R. (eds.) CRYPTO 2012. LNCS, vol. 7417, pp. 517532. Springer, Heidelberg (2012)

43. Micali, S., Reyzin, L.: Physically observable cryptography (extended abstract). In: Naor, M. (ed.) TCC 2004. LNCS, vol. 2951, pp. 278-296. Springer, Heidelberg (2004)

44. Pietrzak, K.: A leakage-resilient mode of operation. In: Joux, A. (ed.) EUROCRYPT 2009. LNCS, vol. 5479, pp. 462-482. Springer, Heidelberg (2009)

45. Ristenpart, T., Tromer, E., Shacham, H., Savage, S.: Hey, you, get off of my cloud: Exploring information leakage in third-party compute clouds. In: Al-Shaer, E., Jha, S., Keromytis, A.D. (eds.) ACM CCS 2009, pp. 199-212. ACM Press (November 2009)

46. Rothblum, G.N.: How to compute under $\mathcal{A C}^{0}$ leakage without secure hardware. In: Safavi-Naini, R., Canetti, R. (eds.) CRYPTO 2012. LNCS, vol. 7417, pp. 552-569. Springer, Heidelberg (2012) 
47. Suh, G.E., Clarke, D.E., Gassend, B., van Dijk, M., Devadas, S.: AEGIS: architecture for tamper-evident and tamper-resistant processing. In: Proceedings of the 17th Annual International Conference on Supercomputing, ICS 2003, pp. 160-171 (2003)

48. Vasudevan, A., McCune, J.M., Newsome, J., Perrig, A., van Doorn, L.: CARMA: A hardware tamper-resistant isolated execution environment on commodity x86 platforms. In: Youl Youm, H., Won, Y. (eds.) ASIACCS 2012, pp. 48-49. ACM Press (May 2012)

49. Yekhanin, S.: Locally decodable codes. Foundations and Trends in Theoretical Computer Science 6(3), 139-255 (2012) 